THEOBROMINE, CATECHIN, TOTAL ANTIOXIDANT CAPACITY AND TOTAL PHENOLIC CONTENT IN REPRESENTATIVE SAMPLES OF BOLIVIAN AMAZONIAN CACAO AND ITS COMPARISON BEFORE AND AFTER THE FERMENTATION PROCESS

\section{DETERMINACIÓN DE TEOBROMINA, CATEQUINA, CAPACIDAD ANTIOXIDANTE TOTAL Y CONTENIDO FENÓLICO TOTAL EN MUESTRAS REPRESENTATIVAS DE CACAO AMAZÓNICO BOLIVIANO Y SU COMPARACIÓN ANTES Y DESPUÉS DEL PROCESO DE FERMENTACIÓN}

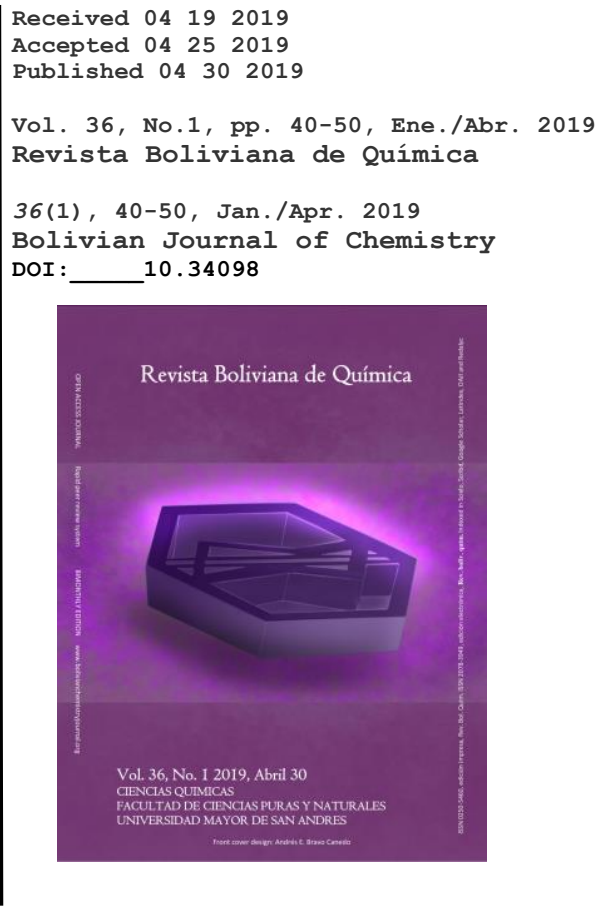

Jimena Ortiz S. ${ }^{1}$, Milenka Chungara ${ }^{1}$, Gabriela Ibieta ${ }^{1}$, Isabel Alejo ${ }^{1}$, Leslie Tejeda ${ }^{1}$, Carmelo Peralta ${ }^{2}$, Enzo Aliaga-Rossel ${ }^{3}$, Patricia Mollinedo ${ }^{1}$, J. Mauricio Peñarrieta ${ }^{1}$

${ }^{1}$ Laboratorio de Químicia de Alimentos, Carrera de Ciencias Químicas, Facultad de Ciências Puras y Naturales FCPN, Universidad Mayor de San Andrés UMSA, P.O. Box 303, Calle Andrés Bello s/n, Ciudad Universitaria Cota Cota, phone +59122792238 , La Paz, Bolivia,

${ }^{2}$ Centro de Investigación y Promoción del Campesinado CIPCA, P. O. Box 5854, Calle Claudio Peñaranda 2706, Sopocachi, La Paz- Bolivia

${ }^{3}$ SWEBOL AB Biotech SRL. Avenida del Escultor 22 Cota Cota, Teléfono +59172078977, La Paz, Bolivia. Scheelevägen 2222363 Lund, Sweden, www.swebol.com, enzo@swebol.com

Keywords: Theobromine, Amazonian cacao, FRAP, ABTS, TAC, TPH, PCA.

Palabras clave: Teobromina, Cacao amazónico, FRAP, ABTS, TAC, TPH, PCA.

\title{
ABSTRACT
}

The Bolivian Amazonian Cocoa (Theobroma cacao) it's a species that grows naturally in the forests, is characterized by its unique flavor, therefore, during three consecutive years it has been recognized and selected among the best cocoas in the world in the international "Salon du Chocolat" Paris France. These characteristics are directly related to the content of chemical compounds such as polyphenols and methylxanthines. 
REVISTA BOLIVIANA DE QUÍMICA

ISSN 0250-5460 Rev. Bol. Quim. Paper edition

ISSN 2078-3949 Rev, boliv, quim. Electronic edition

Jimena Ortiz S. et al. RBQ VoI. 36, No.1, pp. 40-50, 2019

In this research, the chemical functional characteristics of the Bolivian Amazonian cacao, both wild and produced in agroforestry plots from the Amazonian region of Bolivia were determined. Theobromine a methylxanthine and catechin a phenol compound, were quantified by HPLC high-performance liquid chromatography, total antioxidant activity (TAC) by the ABTS and FRAP methods and the total polyphenol content (TPH), in samples of cacao beans before and after the fermentation process.

The values obtained in this study are relevant and this information will serve to highlight the unique characteristics of the Bolivian Amazonian cacao, increasing its value.

\section{*Corresponding author: jimena.ortiz.s@gmail.com}

\section{RESUMEN}

El cacao Amazónico Boliviano silvestre (Theobroma cacao) es una especie que crece de forma natural en los bosques, se caracteriza por ser único en sabor y aroma dentro de los grupos de cacaos más finos, por este motivo, y durante tres años consecutivos ha sido reconocido y seleccionado en el evento internacional "Salón du Chocolat" Paris Francia, entre los mejores cacaos del mundo, caracterizado por su aroma y sabor. Estas características son directamente relacionadas con el contenido de compuestos químicos tales como los polifenoles y metilxantinas.

En la presente investigación se determinaron las características funcionales químicas del cacao Amazónico Boliviano tanto silvestre como cultivadas en parcelas agroforestales de la región amazónica de Bolivia. Se cuantificaron teobromina y catequina por cromatografía líquida de Alta resolución HPLC, la actividad antioxidante total (TAC) por por los métodos ABTS y FRAP y el contenido total de polifenoles (TPH), en muestras, de grano de cacao antes y después del proceso de fermentación.

Los valores obtenidos en este estudio son relevantes y esta información servirá para realzar las características que hacen especial al cacao Amazónico Boliviano, incrementando además su valor.

\section{INTRODUCCIÓN}

EL cacao amazónico boliviano (Theobroma cacao), es un producto que crece de forma natural y silvestre en los bosques amazónicos del país, pero también estas variedades han sido cultivadas y es colectada y utilizada en diferentes regiones del país. Es distribuido en el norte del departamento de La Paz, Santa Cruz, Pando además de los departamentos de Beni y Cochabamba [1]. El cacao es parte importante de la cultura de muchos pueblos originarios de estos departamentos, siendo además un importante aporte económico a las familias. El interés nacional y mundial del cacao con alto valor nutritivo, con propiedades organolépticas únicas, como ser aroma y sabor, que le da cualidades gastronómicas importantes, ha ido incrementando el volumen de producción en todas las regiones extendiendo la demanda a nivel mundial.

El gran consumo mundial de productos derivados del cacao como es el chocolate, la cocoa (cacao soluble en polvo) y otros, han convertido este alimento en un componente en la dieta cotidiana. Su sabor y aroma únicos son atribuidos al contenido de compuestos fenólicos entre los cuales se destacan ( \pm )-catequina y (-)-epicatequina por su efecto antioxidante [2].

Es por la presencia de esos polifenoles que se reconocen los beneficios en la salud, como el control de enfermedades cardiovasculares, inflamatorias y las derivadas del estrés celular, sin embargo, los productos de cacao no solo son ricos en polifenoles, también son ricos en otros compuestos químicos como las metilxantinas, que representan alrededor de 3,2 \% de la composición del cacao [3]. Las principales metilxantinas de cacao son la teobromina $(3,7 \%$ sobre una base sin grasa) y cafeína (alrededor de $0,2 \%)$. La posible sinergia de las interacciones entre los flavonoides y metilxantinas, todavía no se han comprobado, pero se debe considerar el aporte de las propiedades beneficiosas para la salud de la teobromina, tales como las propiedades estimulantes y antidepresivas en los productos de cacao [4].

Como fue mencionado, el cacao es en particular rico en polifenoles, representando entre 12 y $18 \%$ del peso seco de los granos, y se encuentran fuertemente asociados con la actividad antioxidante y con las características organolépticas de los productos elaborados a partir del cacao [4].

Los polifenoles en los granos de cacao se encuentran mayormente en las células pigmentarias de los cotiledones y son los responsables de los colores variados del fruto, dependiendo de la cantidad de antocianinas (flavonoides que producen color en los vegetales), los frutos pueden presentar un color rojizo muy intenso [5]. En los frutos de cacao

Downloadable from: Revista Boliviana

de Química. Volumen 36 Nº1. Año 2019

http://www.bolivianchemistryjournal.org, http://www.scribd.com/bolivianjournalofchemistry 
REVISTA BOLIVIANA DE QUÍMICA

ISSN 0250-5460 Rev. Bol. Quim. Paper edition ISSN 2078-3949 Rev, boliv, quim. Electronic edition

Jimena Ortíz S. et al. RBQ VoI. 36, No.1, pp. 40-50, 2019

se pueden distinguir 3 grupos de polifenoles: catequinas o flavan-3-oles $(37 \%)$, antocianinas $(4 \%)$ y proantocianidinas $(58 \%)$ [2]. Los principales flavonoles presentres en el cacao son la (+)-catequina y (-) epicatequina con un máximo de hasta $35 \%$ del contenido total de polifenoles. También se han encontrado en cantidades menores, (+)-galocatequina y (-) epigalocatequina.

Una de las etapas previas e importantes para la fabricación de chocolate es la fermentación de los granos de cacao, la cual se lleva a cabo en el lugar de la cosecha o colecta, donde por varios días se dejan los granos de cacao a temperaturas controladas mayores a la ambiental. Durante la fermentación se producen importantes cambios bioquímicos responsables de la formación de los precursores del aroma y sabor de chocolate, lo que determina su calidad física y química como chocolate [6]. Entre estos cambios se puede mencionar el desarrollo del cambio de color o pardeamiento del grano, a partir de la descomposición de los compuestos fenólicos, lo cual es un indicativo final de la fermentación del grano de cacao. Además, los contenidos finales de polifenoles, alcaloides (cafeína y teobromina) y acidez (en especial el contenido de ácido acético) [7], son los indicadores de la calidad organoléptica del cacao fermentado.

Los alcaloides están asociados con el sabor amargo del cacao. Su concentración depende de la variedad y se modifica con el procesamiento, particularmente durante el fermentado. Los polifenoles junto con otras moléculas son responsables, de la astringencia del chocolate, además de las propiedades antioxidantes deseables por los consumidores.

La fermentación del cacao además de complejas reacciones enzimáticas también involucra la degradación microbiana en la pulpa. Los azúcares simples son metabolizados por levaduras y bacterias lácticas en condiciones anaeróbicas, para producir etanol y ácido láctico respectivamente [8].

El sabor y aroma de los granos de cacao fueron las principales razones que promovieron su domesticación y uso como alimento por los pueblos pre coloniales de América. Estos parámetros actualmente son importantes para determinar la calidad sensorial y diferenciar al cacao entre fino y a granel. [9].

La presente investigación, determina el contenido de antioxidantes totales, por los métodos ABTS, FRAP y el contenido Fenólico total por el método de Folin - Ciocalteu y teobromina, por el método de HPLC, antes y después del proceso de fermentación en muestras de cacao amazónico boliviano.

\section{EXPERIMENTAL}

\section{Obtención de las muestras}

Las muestras de Cacao Amazónico (Theobroma cacao) fueron obtenidas en las zonas de acción del Centro de Investigación y Promoción del Campesinado CIPCA, que comprenden los municipios: Gonzalo Moreno y Riberalta en el departamento de Pando, los municipios de Baures y San Ignacio de Mojos del departamento del Beni, Bolivia. Se tomaron muestras tanto en sistemas agroforestales (SAF) como en producción silvestre. En las SAFs se consideró aquellas parcelas accesibles y representativas además de aquellas muestras que fueron ganadoras de premios internacionales.

\section{Reactivos}

El reactivo Folin-Ciocalteu, ácido gálico, carbonato de sodio, ABTS [2,2'-azino-bis (3-etilbenzotiazolina-6-ácido sulfónico)], persulfato de potasio, Trolox (6-hidroxi-2,5,7,8-tetrametilchroman-2-ácido carboxilico, 97\%), TPTZ (2,4,6-tripiridil-s-triazina), cloruro férrico, fosfato monobásico de sodio, cloruro de sodio, Catequina y Teobromina fueron obtenidos de Sigma-Aldrich (St. Louis, USA), metanol absoluto de J.T. Baker (México DF, México) y metanol de Merck (Darmstadt, Alemania).

\section{Extracción de las Muestras}

Para realizar la extracción de las muestras, se tomó $0,1 \mathrm{~g}$ de cada muestra. Cada muestra fue extraída con $1 \mathrm{ml}$ de solución de Etanol - Agua producida en un sistema Milli-Q (Millipore Corp., Billerica, MA, EUA.) (90\% etanol), posteriormente se llevó al ultrasonido por $15 \mathrm{~min}$ a $0{ }^{\circ} \mathrm{C}$ en un equipo Elmasonic model S60H (Singen, Alemania), luego fueron centrifugadas a 12000 revoluciones por 2 minutos a $4^{\circ} \mathrm{C}$ En una centrífuga CentrisartA-14 - Sartorius

Downloadable from: Revista Boliviana

de Química. Volumen 36 Nº1. Año 2019

http://www.bolivianchemistryjournal.org, http://www.scribd.com/bolivianjournalofchemistry 
ISSN 2078-3949 Rev, boliv, quim, Electronic edition

(Göttingen, Alemania). El sobrenadante fue separado y almacenado a $-20^{\circ} \mathrm{C}$, el precipitado fue secado a $45^{\circ} \mathrm{C}$ por un periodo de 24 horas, posteriormente pesado. [10]. La Tabla 1 describe la procedencia de las muestras, los códigos asignados.

Tabla 1: Muestras de Cacao; se detalla el municipio, la comunidad y la procedencia de la planta

\begin{tabular}{|c|c|c|c|}
\hline Código & Municipio & Comunidad & $\begin{array}{c}\text { Procedencia de } \\
\text { Planta }\end{array}$ \\
\hline$R V-21 *$ & Gonzalo Moreno & $\begin{array}{c}\text { Contravaricia-lugar río } \\
\text { verde* }\end{array}$ & SAF \\
\hline$M I R-17 *$ & Gonzalo Moreno & Miraflores* & SAF \\
\hline$C V-30 *$ & Gonzalo Moreno & Contravaricia* & Silvestre \\
\hline MIR-19* & Gonzalo Moreno & Miraflores* & SAF \\
\hline$R V-22$ & Gonzalo Moreno & $\begin{array}{c}\text { Contravaricia-lugar río } \\
\text { verde }\end{array}$ & SAF \\
\hline$M I R-18$ & Gonzalo Moreno & Miraflores & SAF \\
\hline$C V-28$ & Gonzalo Moreno & Contravaricia & Silvestre \\
\hline$R V-24$ & Gonzalo Moreno & $\begin{array}{c}\text { Contravaricia-lugar río } \\
\text { verde }\end{array}$ & SAF \\
\hline$R V-23$ & Gonzalo Moreno & $\begin{array}{c}\text { Contravaricia-lugar río } \\
\text { verde }\end{array}$ & SAF \\
\hline$C V-31$ & Gonzalo Moreno & Contravaricia & Silvestre \\
\hline$C V-32$ & Gonzalo Moreno & Contravaricia & Silvestre \\
\hline$C V-29$ & Gonzalo Moreno & Contravaricia & Silvestre \\
\hline$M I R-16$ & Gonzalo Moreno & Miraflores & SAF \\
\hline MIR-20 & Gonzalo Moreno & Miraflores & SAF \\
\hline$R V-25$ & Gonzalo Moreno & $\begin{array}{c}\text { Contravaricia-lugar río } \\
\text { verde }\end{array}$ & SAF \\
\hline$N A Z-3$ & Riberalta & Nazareth & Silvestre \\
\hline$N A Z-2$ & Riberalta & Nazareth & Silvestre \\
\hline$S J-12$ & Riberalta & Siete de Julio & Silvestre \\
\hline$S J-15$ & Riberalta & Siete de Julio & Silvestre \\
\hline$N A Z-7$ & Riberalta & Nazareth & SAF \\
\hline$N A Z-8$ & Riberalta & Nazareth & SAF \\
\hline$S J-11$ & Riberalta & Siete de Julio & Silvestre \\
\hline$N A Z-4$ & Riberalta & Nazareth & Silvestre \\
\hline$N A Z-5$ & Riberalta & Nazareth & Silvestre \\
\hline$S J-9 *$ & Riberalta & Siete de Julio* & SAF \\
\hline$N A Z-6$ & Riberalta & Nazareth & SAF \\
\hline SJ-10 * & Riberalta & Siete de Julio* & SAF \\
\hline$S J-14$ & Riberalta & Siete de Julio & Silvestre \\
\hline$N A Z-1$ & Riberalta & Nazareth & Silvestre \\
\hline$S J-13$ & Riberalta & Siete de Julio & Silvestre \\
\hline$S C-8$ & Baures & Catiyene & Silvestre \\
\hline$C T-13$ & Baures & Catiyene & Silvestre \\
\hline$S P-29$ & Baures & San Pablo & Silvestre \\
\hline$I T V-23$ & Baures & Catiyene & Silvestre \\
\hline$E A R \_28$ & San Ignacio Moxos & Santa Rosa & Silvestre \\
\hline$S R A-8$ & San Ignacio Moxos & Santa Rosa & Silvestre \\
\hline$E A R \_33$ & San Ignacio Moxos & San Miguel de Matire & Silvestre \\
\hline
\end{tabular}

Downloadable from: Revista Boliviana 
REVISTA BOLIVIANA DE QUÍMICA

ISSN 0250-5460 Rev. Bol. Quim. Paper edition

ISSN 2078-3949 Rev, boliv, quim. Electronic edition

Jimena Ortíz S. et al. RBQ VoI. 36, No.1, pp. 40-50, 2019

Análisis de antioxidantes
Received 04192019 36(1); Jan./Apr. 2019

Accepted 00002019

Published 0430 2019; DOI: 10.34098

La capacidad antioxidante, fue determinada por dos métodos, el método de reducción férrica del poder antioxidante con sus siglas en inglés FRAP y el método basado en la decoloración del radical del ácido 2,2'-azino-bis (3etilbenzotiazolin-6-sulfonico), conocido como ABTS.

El método FRAP evalúa la capacidad antioxidante de una muestra de acuerdo a su capacidad para reducir el complejo amarillo de Fe3+-TPTZ (2, 4, 6-tripiridil-s-triazina) al complejo azul de Fe2+-TPTZ. Este último complejo tiene un máximo de absorbancia a una longitud de onda entre 590-595 nm [11].

Para la lectura de las muestras se utilizaron $900 \mu \mathrm{L}$ de solución FRAP, $30 \mu \mathrm{L}$ de muestra y $120 \mu \mathrm{L}$ de agua destilada. La solución FRAP está compuesta por $25 \mathrm{ml}$ de solución buffer ácido acético-acetato de sodio (pH 3,6), $2,5 \mathrm{ml}$ de solución $10[\mathrm{mM}] \mathrm{TPTZ}$ diluida con $\mathrm{HCl} 40[\mathrm{mM}]$ y solución $20[\mathrm{mM}] \mathrm{FeCl}_{3}$. Se determinó la absorbancia a una longitud de onda de $593 \mathrm{~nm}$ en un lector multi-modal Cytation 3, marca BioTek (Vermont, USA). Para cada lectura se tuvo en cuenta la lectura de la absorbancia de la muestra testigo. La absorbancia final de las muestras se comparó con la curva estándar Trolox (100-1000 $\mu \mathrm{mol} / \mathrm{L})$ disuelto con etanol al 96\%. [12].

El método ABTS se fundamenta en la cuantificación de la decoloración del radical $\mathrm{ABTS}^{+}$, debido a su reducción a ABTS por la acción de antioxidantes. El radical catiónico $\mathrm{ABTS}^{+}$es un cromóforo verde azulado que absorbe a una longitud de onda de $734 \mathrm{~nm}$ y se genera por una reacción de oxidación del ABTS (2,2'-azino-bis- (3etil benzotiazolin-6-sulfonato de amonio) con persulfato de potasio. De esta manera el grado de decoloración como porcentaje de inhibición del radical $\mathrm{ABTS}^{+}$está determinado en función a la concentración. En la evaluación se utilizaron $100 \mu \mathrm{L}$ de la muestra y $1 \mathrm{~mL}$ de la solución del radical $\mathrm{ABTS}^{+}$. La solución del radical $\mathrm{ABTS}^{+}$se preparó mezclando $5 \mathrm{ml}$ de solución ABTS $7[\mathrm{mM}]$ con $88 \mu \mathrm{L}$ de solución de persulfato de potasio 140 [mM], posteriormente se diluyó en buffer ácido acético-acetato de sodio $(\mathrm{pH}=5)$ hasta obtener una absorbancia de 0,7 aproximadamente. Las mediciones se realizaron a una longitud de onda de $734 \mathrm{~nm}$ a los 3 min de reacción y a temperatura ambiente. Se utilizó un lector multi-modal Cytation 3, marca BioTek (Vermont, USA). Los resultados se expresaron mediante la construcción de una curva patrón usando como antioxidante trolox $(0-200 \mu \mathrm{mol} / \mathrm{L})$ [12].

\section{Determinación de contenido de fenoles totales}

El análisis de fenoles totales se realizó por el método de Folin-Ciocalteu, que se basa en que los compuestos fenólicos reaccionan con el reactivo de Folin-Ciocalteu en medio básico dando lugar a una coloración azul para luego ser determinada espectrofotométricamente a $765 \mathrm{~nm}$.[11] Este reactivo contiene una mezcla de wolframato sódico y molibdato sódico en ácido fosfórico, el ácido fosfomolibdo túngstico formado, de color amarillo, al ser reducido por los grupos fenólicos da lugar a un complejo de color azul intenso, cuya intensidad es la que se determina para evaluar el contenido de polifenoles. Para la determinación de Fenoles Totales se mezcló $50 \mu \mathrm{L}$ de muestra, $1000 \mu \mathrm{L}$ solución Folin \& Ciocalteu (1:10 V/V) y $500 \mu \mathrm{L}$ de carbonato de sodio (7,5\%) [11]. La mezcla se homogenizo y calentó a 45 ${ }^{\circ} \mathrm{C}$ por 30 minutos, se midió la absorbancia a $765 \mathrm{~nm}$ en un lector multi-modal Cytation 3, marca BioTek (Vermont, EUA). La absorbancia final de cada muestra se comparó con una curva estándar de calibración de ácido gálico (40$200 \mathrm{mg} / \mathrm{l})$ [12].

\section{Análisis de Teobromina y Catequina por HPLC}

Para realizar el análisis por HPLC, previamente se realizó una hidrólisis ácida de las muestras, utilizamos $\mathrm{HCl}$ al 20 $\%$ y extractos de las muestras diluidas $1: 10$. Se agregó a $500 \mu \mathrm{L}$ de muestra, $500 \mu \mathrm{L}$ de $\mathrm{HCl}$, la hidrolisis se llevó a cabo por 2 horas a una temperatura de $90{ }^{\circ} \mathrm{C}$. [10].

Este análisis se realizó por Cromatografía Liquida de Alta Eficacia de fase reversa. Para esto se utilizó el equipo HPLC Agilent (Palo Alto, CA, EUA) series1100. La columna utilizada para la determinación de Teobromina y Catequina fue una Zorbax, Agilent Eclipse Plus C18, $150 \mathrm{~mm}$, de fase inversa, protegida por una pre-columna de 10 $\mathrm{mm}$. El caudal empleado fue de $0,7 \mathrm{~mL} / \mathrm{min}$ y se inyectó un volumen de $20 \mu \mathrm{L}$ por muestra. La fase móvil fue un solvente de sistema binario que consistió en: $1 \%$ ácido acético / agua (A) y metanol (B). El gradiente utilizado fue 20 $\%$ de (B) a los 0 minutos, 30\% de (B) después de 5 minutos, $40 \%$ de (B) después de 10 minutos, $50 \%$ de (B) después de 15 minutos, $65 \%$ de (B) a los 17 minutos, $80 \%$ de (B) después de 19 minutos y 20\% de (B) después de 15 minutos, los tiempos de retención y los cromatogramas fueron comparados con los de un estándar puro de 
REVISTA BOLIVIANA DE QUÍMICA

ISSN 0250-5460 Rev. Bol. Quim. Paper edition ISSN 2078-3949 Rev, boliv, quim, Electronic edition

Jimena Ortiz S. et al. RBQ VoI. 36, No.1, pp. 40-50, 2019

teobromina y catequina para la identificación del pico correspondiente en las muestras. [10], el tiempo de retención para la teobromina es de 7,6 min y el tiempo de retención para la catequina es de 12,7 minutos.

\section{Análisis estadístico}

Para los ensayos de Fenoles Totales, FRAP y ABTS se realizaron lecturas por triplicado durante dos diferentes días Todos los cálculos fueron hechos usando hojas de datos en el programa Excel. Los valores fueron expresados como el promedio de las seis lecturas \pm la desviación estándar.

Para evaluar la diferencia estadística de los valores de la capacidad antioxidante total de las muestras por municipio, antes y después del proceso de fermentación del cacao, se empleó la prueba de t de student, y la Prueba de Shapiro - Wilk con un nivel de significancia del 5\%. El análisis se realizó utilizando el software IBM SPSS STATISTICS 22. (NY. EUA).

Además, se realizó el análisis multivariable de Componente principales utilizando el paquete estadístico $\mathrm{R}$ versión R version 3.5.1 (R Foundation for Statistical Computing, Vienna, Austria).

\section{RESULTADOS}

Los valores obtenidos para la capacidad antioxidante total, el contenido de compuestos fenólicos y la concentración de teobromina y catequina de las muestras seleccionadas se encuentran descritos en la Tabla 1.

\section{Capacidad antioxidante Total (TAC)}

Los resultados de la capacidad antioxidante total obtenidos por el método de ABTS del municipio de Gonzalo Moreno se encuentran en un rango de 130,3 - 15,9 $\mu \mathrm{mol} / \mathrm{g}$ en muestras sin fermentar y $20,6-3,5 \mu \mathrm{mol} / \mathrm{g}$ en muestras fermentadas, para el municipio de Riberalta se tiene datos en el rango de 78-28,1 $\mu \mathrm{mol} / \mathrm{g}$ en muestras sin fermentar y 13,3 - 5,2 $\mu \mathrm{mol} / \mathrm{g}$ en muestras fermentadas, para el municipio de Baures $126,4-43,2 \mu \mathrm{mol} / \mathrm{g}$ en muestras sin fermentar y 5,79-3,15 en muestras fermentadas, y para el municipio de San Ignacio de Mojos se tiene un rango de $16,1-8,6 \mu \mathrm{mol} / \mathrm{g}$ en muestras sin fermentar y un promedio de $5,5 \mu \mathrm{mol} / \mathrm{g}$ en muestras fermentadas.

El contenido de la capacidad antioxidante obtenido por el método FRAP, se encuentra en un rango de 206 $6,30 \mu \mathrm{mol} / \mathrm{g}$ en muestras sin fermentar y $24,5-1,87 \mu \mathrm{mol} / \mathrm{g}$ en muestras fermentadas del municipio de Gonzalo Moreno, en el municipio de Riberalta obtuvimos un rango de 133,9 - 39,1 $\mu \mathrm{mol} / \mathrm{g}$ en muestras sin fermentar y 6,9$1,71 \mu \mathrm{mol} / \mathrm{g}$ en muestras fermentadas, para el municipio de Baures tenemos un rango de $106-54,9 \mu \mathrm{mol} / \mathrm{g}$ en muestras sin fermentar y 7,2 - 2,06 $\mu \mathrm{mol} / \mathrm{g}$ en muestras fermentadas, y para el municipio de San Ignacio de Mojos tenemos un rango de 6,7-6 $\mu \mathrm{mol} / \mathrm{g}$ en muestras sin fermentar y un promedio de $2,5 \mu \mathrm{mol} / \mathrm{g}$ en muestras fermentadas.

Los valores obtenidos por ambos métodos son similares en las muestras no fermentadas, siendo los valores más altos los obtenidos en las muestras del municipio de Gonzalo Moreno del departamento de Pando.

\section{Contenido de Fenoles Totales (TPH)}

Los resultados obtenidos del contenido de fenoles totales, se encuentran en un rango de $159-10,2 \mathrm{mmol} / \mathrm{g}$ en muestras no fermentadas, y 19,1 - 0,88 mmol/g en muestras fermentadas del municipio de Gonzalo Moreno, el rango obtenido para las muestras de Riberalta se encuentran entre 222,6-20,9 mmol/g para muestras sin fermentar y 4,66 $0,81 \mathrm{mmol} / \mathrm{g}$ en muestras fermentadas, para el municipio de Baures tenemos $97-25,1 \mathrm{mmol} / \mathrm{g}$ en muestras sin fermentar y 13,9 - 1,05 mmol/g en muestras fermentadas y para el municipio de San Ignacio de Mojos tenemos los datos en un rango de $6,8-6,5 \mathrm{mmol} / \mathrm{g}$ en muestras sin fermentar y un promedio de 1,6 $\mathrm{mmol} / \mathrm{g}$ en muestras fermentadas. El mayor valor de contenido fenólico se encuentra en muestras del municipio de Riberalta, en muestras no fermentadas y en muestras fermentadas el valor más alto se encontró en el municipio de Baures.

Concentración de Teobromina y catequina.

Downloadable from: Revista Boliviana 
Para la identificación de teobromina y catequina presentes en las muestras de cacao, se utilizó el equipo de HPLC, previamente se realizó una hidrólisis ácida a las muestras, utilizando el método como se encuentra descrito por Tejeda et al. [10]. Se realizaron curvas patrón de teobromina y catequina, con las concentraciones de 20, 40, 60, 80 y 100 ppm, y obtuvieron las mejores condiciones cromatográficas, según el método [10]. El tiempo de retención para el estándar de teobromina a 80 ppm es de 7,6 minutos y el espectro UV presenta absorción a una longitud de onda de $280 \mathrm{~nm}$.

En la figura 1, se presenta el cromatograma del estándar de teobromina y el cromatograma de una muestra, obteniendo así la concentración de teobromina de cada una de las muestras expresadas en la tabla 1.

Los datos obtenidos de concentración de teobromina para el municipio de Gonzalo Moreno se encuentran entre un rango de 3,10 - 0,06 mg/g en muestras no fermentadas y $1,8-0,13 \mathrm{mg} / \mathrm{g}$ en muestras fermentadas, en el municipio de Riberalta se obtuvo un rango de $3,95-0,07 \mathrm{mg} / \mathrm{g}$ en muestras no fermentadas y $8,43-0,32 \mathrm{mg} / \mathrm{g}$ en muestras fermentadas, para el municipio de Baures se tiene 5,78 - 3,94 mg/g en muestras no fermentadas y 1,14 $0,906 \mathrm{mg} / \mathrm{g}$ en muestras fermentadas, y en el municipio de San Ignacio de Mojos se tiene un rango de 8,20 - 4,37 mg/ $\mathrm{g}$ en muestras sin fermentar .

\section{DISCUSIONES}

Se analizaron muestras de cuatro municipios de la región amazónica de Bolivia, Gonzalo Moreno en Pando y Riberalta, Baures y San Ignacio de Mojos en Beni, se tomaron en cuenta las muestras más representativas de estos lugares, antes y después del proceso de fermentación.

Los resultados obtenidos de la Capacidad Antioxidante total por el método ABTS de granos de cacao fermentados y sin fermentar de los municipios Gonzalo Moreno y Baures, se encuentran dentro del rango de datos reportados en granos de cacaos colombianos fermentados y no fermentados según bibliografía [13,14]. Sin embargo, los valores obtenidos para las muestras de los municipios de Riberalta y San Ignacio de Mojos en muestras sin fermentar están por debajo de los valores reportados en bibliografía [15].

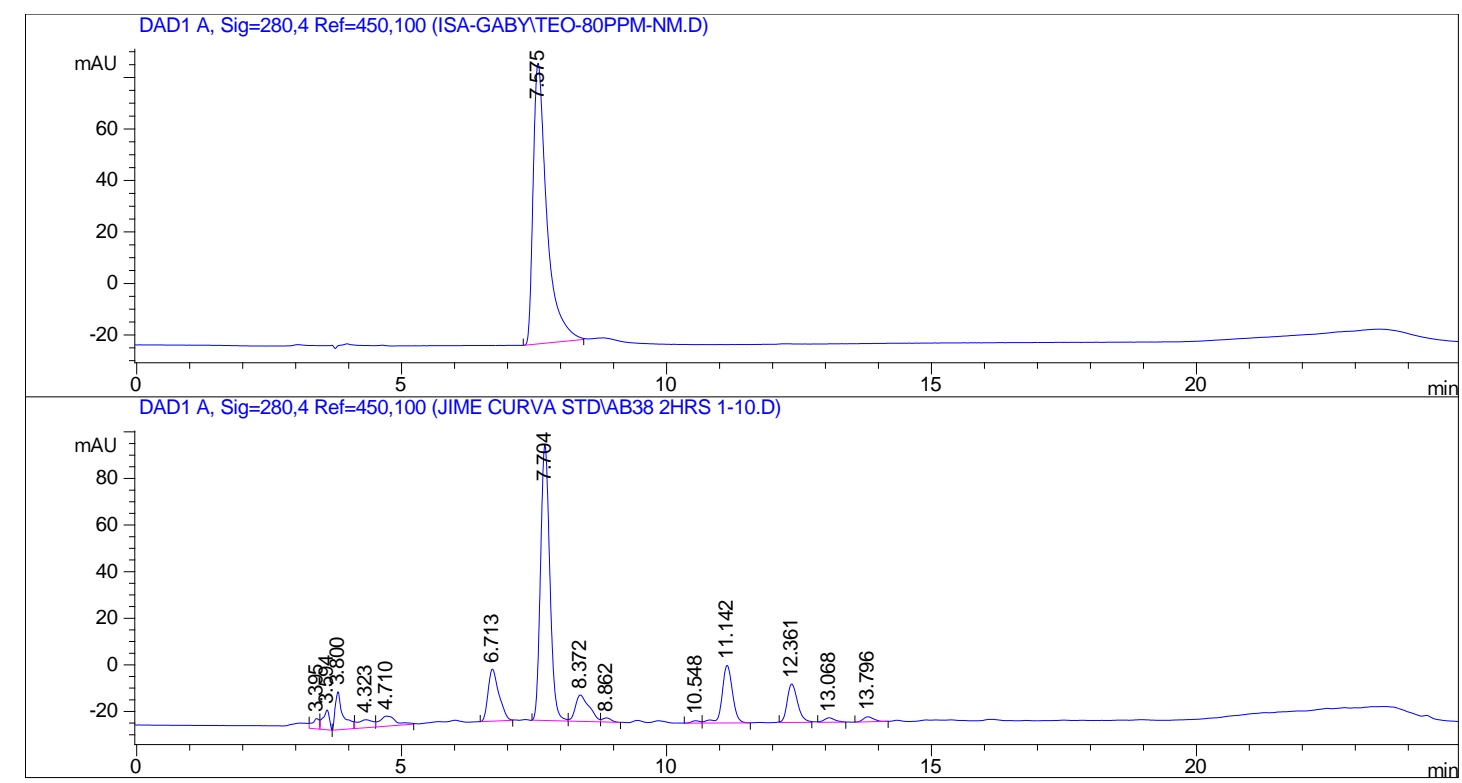

Figura 1. Resultados del HPLC, cromatograma del estándar de Theobromina, comparado con el cromatograma de la muestra SC8 del municipio de Baures 
Jimena Ortíz S. et al. RBQ Vol. 36, No.1, pp. 40-50, 2019

Los valores obtenidos por FRAP en las muestras sin fermentar de todos los municipios, se encuentran dentro el rango reportado por bibliografía, mientras que los valores de las muestras fermentadas están por debajo del rango del mismo. [16,17].

El contenido de fenoles totales (TPH) se encuentra en el rango reportado por bibliografía en muestras fermentadas y sin fermentar [4] y [15].

El presente trabajo confirma que la actividad antioxidante total en cacao, decrece luego del proceso de fermentación.

Para poder evaluar si la disminución de la capacidad antioxidante es significativa después del proceso de fermentación, se realizó la prueba $t$ de student. Los resultados de esta prueba mostraron una significancia $\mathrm{p}<0,05$ aceptando esta hipótesis donde la diferencia de medias es estadísticamente significativa para los métodos ABTS y FRAP.

En cuanto a los resultados de la concentración de teobromina y catequina en las muestras de cacao fermentadas, la concentración de catequina de los municipios de Gonzalo Moreno y Riberalta está dentro de los valores obtenidos en otros estudios revisados en la bibliografía [2] y los valores de teobromina se encuentran en mayor porcentaje en muestras no fermentadas, en comparación de datos reportados en un estudio realizado en Colombia y Ecuador, lo que sugiere que estos cacaos son más amargos, ya que el contenido de teobromina, está relacionado con el sabor amargo. [4] y [6].

El contenido de Teobromina puede ser influenciado por el factor localidad, la variación de, estado de la planta puede ser cultivado, silvestre o en SAF, y el proceso de fermentación uniforme, encontrando de igual manera sobre el rango de valores de cacao del presente estudio. [18] y [19].

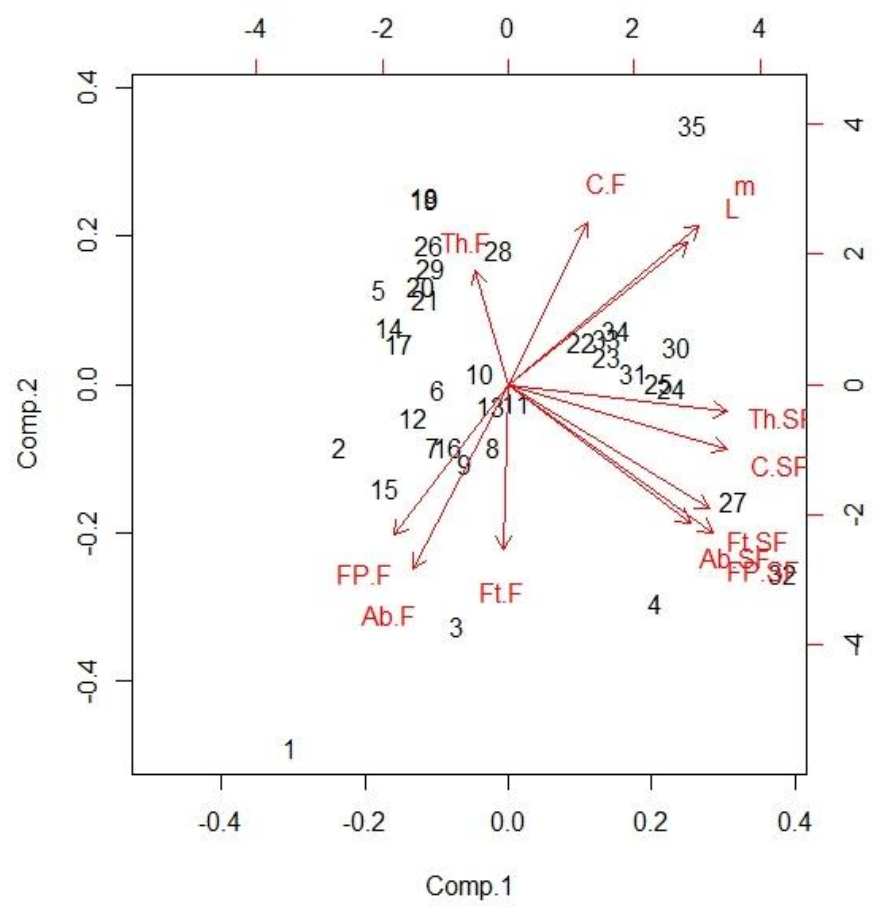

Figura 2. Biplot, PCA (Principal Component Analysis) Análisis de componentes principales, donde: Ab.F= Método ABTS, en muestras fermentadas, Ab.SF= Método ABTS, en muestras sin fermentar, FtF. = Fenoles totales, en muestras fermentadas, Ft. $S F .=$ Fenoles totales en muestras sin fermentar, Th.F. $=$ Concentración de Theobromina en muestras fermentadas, Th.SF. $=$ Concentración de Theobromina en muestras sin fermentar, CF = Concentración de Catequina en muestras fermentadas, C.SF.$=$ Concentración de Catequina en muestras sin fermentadas, FP. F= Método FRAP en muestras fermentadas, FP.SF. = Método FRAP en muestras sin fermentar, $m=$ Muestra, l= lugar.

En el análisis de componentes principales se observa que los datos medidos en las muestras se agrupan de manera simultánea principalmente por municipio, formando dos clusters, el primero de muestras 1-15 que corresponden al municipio de Gonzalo Moreno y el segundo a los restantes municipios.

$$
\begin{aligned}
& \begin{array}{c}
\text { Downloadable from: Revista Boliviana } 47 \quad \text { de Química. Volumen } 36 \text { N}^{\circ} 1 \text {. Año } 2019 \\
\text { http://www.bolivianchemistryjournal.org, }
\end{array} \underline{\text { http://www.scribd.com/bolivianjournalofchemistry }}
\end{aligned}
$$


REVISTA BOLIVIANA DE QUÍMICA

ISSN 0250-5460 Rev. Bol. Quim. Paper edition

ISSN 2078-3949 Rev, boliv, quim. Electronic edition

Jimena Ortíz S. et al. RBQ VoI. 36, No.1, pp. 40-50, 2019

El efecto de la fermentación sobre los cacaos se observa de manera uniforme en el municipio de Gonzalo Moreno (muestras de $1-15$ ). Esto se puede observar en el gráfico que se agrupan cerca, los parámetros antioxidantes ABTS y FRAP, después de la fermentación [20] conformando estas muestras un cluster, mientras que las muestras de los demás municipios son representadas por la actividad antioxidantes antes de la fermentación y se agrupan de manera menos homogénea [21-23].

El análisis PCA también sugiere que la actividad antioxidante y el contenido de compuesto fenólicos después del fermentado pueden ser considerados como parámetros para determinar la calidad de la fermentación, aroma y sabor de los granos de cacao, ya que las muestras 1 - 4 ganadoras al mejor grano de cacao a nivel internacional, se encuentran cercanas a dichos parámetros en la gráfica.

Esto indica que el proceso de fermentación de forma uniforme es importante para la calidad organoléptica del cacao y que en el municipio de Gonzalo Moreno este proceso destaca de los demás y correlaciona con la calidad de sus productos.

\section{CONCLUSIONES}

Esta investigación, contribuye a la revalorización de los alimentos bolivianos, como es el cacao amazónico, que además es parte de la gran biodiversidad que existe en el país, aportando por primera vez valores e interpretaciones acerca del contenido de polifenoles, la capacidad antioxidante total y la concentración de teobromina, factores directamente relacionados con la calidad, aroma y sabor del cacao.

Los resultados obtenidos muestran que el proceso de fermentación es importante para la calidad organoléptica del cacao y el contenido de compuestos antioxidantes y polifenoles podrían ser indicadores químicos de una buena fermentación, lo cual serviría para la clasificación y selección de cacaos de calidad.

Más estudios son necesarios acerca del origen, la composición y el desarrollo de productos a partir del cacao amazónico boliviano.

\section{AGRADECIMIENTOS}

A la ONG CIPCA (Centro de investigación y promoción al campesinado) por su contribución a este trabajo. A la cooperación sueca - Asdi, por el financiamiento a esta investigación. 
Tabla 2. Resultados de contenido de antioxidantes por los métodos ABTS, FRAP, TPH y concentración de teobromina y catequina en muestras de cacao fermentadas y no fermentadas

\begin{tabular}{|c|c|c|c|c|c|c|c|c|c|c|c|}
\hline \multirow[b]{2}{*}{ Municipio } & \multirow[b]{2}{*}{ Código } & \multicolumn{2}{|c|}{ ABTS } & \multicolumn{2}{|c|}{ FRAP } & \multicolumn{2}{|c|}{ TPH } & \multicolumn{2}{|c|}{ CATECHN } & \multicolumn{2}{|c|}{ THEOBROMNE } \\
\hline & & $\begin{array}{l}\text { Muestras sin } \\
\text { fermentar } \\
(\mathrm{TAC})(\mu \mathrm{md} / \mathrm{g})\end{array}$ & $\begin{array}{l}\text { Muestras } \\
\text { fermentadas } \\
(\mathrm{TAC})(\mu \mathrm{md} / \mathrm{g})\end{array}$ & $\begin{array}{l}\text { Muestras sin } \\
\text { fermentar } \\
(T A C)(\mu \mathrm{md} / \mathrm{g})\end{array}$ & $\begin{array}{l}\text { Muestras } \\
\text { fermentadas } \\
(T A C)(\mu \mathrm{md} / \mathrm{g})\end{array}$ & $\begin{array}{c}\text { Muestras sin } \\
\text { fermentar } \\
\text { (TPH) } \\
(\mathrm{mmol} / \mathrm{g})\end{array}$ & $\begin{array}{c}\text { Muestras } \\
\text { fermentadas } \\
(\mathrm{TPH}) \\
(\mathrm{mmol} / \mathrm{g})\end{array}$ & $\begin{array}{c}\text { Muestras sin } \\
\text { fermentar } \\
(\mathrm{mg} / \mathrm{g})\end{array}$ & $\begin{array}{c}\text { Muestras } \\
\text { Fermentadas } \\
(\mathrm{mg} / \mathrm{g})\end{array}$ & $\begin{array}{c}\text { Muestras } \sin \\
\text { fermentar }(\mathrm{mg} / \mathrm{g})\end{array}$ & $\begin{array}{c}\text { Muestas } \\
\text { fermentadas } \\
(\mathrm{mg} / \mathrm{mg})\end{array}$ \\
\hline Gonzalo Moreno * & $R V-21^{*}$ & $15,9 \pm 0,3$ & $12,1 \pm 0,2$ & $6,30 \pm 0,1$ & $24,5 \pm 0,4$ & $10,7 \pm 0,1$ & $19,1 \pm 0,2$ & $S D$ & $S D$ & $0,112 \pm 0,0012$ & $S D$ \\
\hline Gonzalo Moreno * & $M I R-17^{*}$ & SD & $9 \pm 0,1$ & $S D$ & $5,1 \pm 0,2$ & $S D$ & $3,7 \pm 0,1$ & $S D$ & $S D$ & $S D$ & $S D$ \\
\hline Gonzalo Moreno * & $\mathrm{CV}-30^{*}$ & $34,7 \pm 0,5$ & $20,6 \pm 0,4$ & $80 \pm 1$ & $4,8 \pm 0,2$ & $54 \pm 2$ & $3,2 \pm 0,1$ & $1,58 \pm 0,27$ & SD & $0,739 \pm 0,045$ & $S D$ \\
\hline Gonzalo Moreno * & MIR-19* & $130,3 \pm 4,0$ & $11,3 \pm 0,1$ & $206 \pm 6$ & $2,4 \pm 0,1$ & $159 \pm 3$ & $2,1 \pm 0,1$ & $0,55 \pm 0,7$ & $0,56 \pm 0,8$ & $0,82 \pm 0,12$ & $1,368 \pm 1,63$ \\
\hline Gonzalo Moreno & $R V-22$ & SD & $3,5 \pm 0,1$ & $S D$ & $2,3 \pm 0,1$ & $S D$ & $2 \pm 0,1$ & $S D$ & $1,84 \pm 0$ & $S D$ & $1,80 \pm 0,01$ \\
\hline Gonzalo Moreno & MIR-18 & $20,0 \pm 0,1$ & $7,5 \pm 0,1$ & $7,4 \pm 0,1$ & $2,75 \pm 0,05$ & $10,2 \pm 0,1$ & $1,73 \pm 0,01$ & $0,82 \pm 0,08$ & $S D$ & $0,04 \pm 0,02$ & $S D$ \\
\hline Gonzalo Moreno & CV-28 & $32,5 \pm 0,1$ & $5,8 \pm 0,1$ & $58 \pm 2$ & $2,8 \pm 0,1$ & $34,9 \pm 0$ & $1,69 \pm 0,02$ & $S D$ & $S D$ & $S D$ & $0,104 \pm 0,021$ \\
\hline Gonzalo Moreno & $R V-24$ & $47,8 \pm 1,7$ & $9,25 \pm 0,03$ & $52,3 \pm 0,8$ & $2,4 \pm 0,1$ & $45,4 \pm 0,3$ & $1,48 \pm 0,01$ & $4,59 \pm 0,5$ & SD & $0,108 \pm 0,004$ & $S D$ \\
\hline Gonzalo Moreno & $R V-23$ & $60,6 \pm 0,8$ & $5,8 \pm 0,1$ & $75,6 \pm 0,3$ & $2,13 \pm 0,03$ & $35,5 \pm 0,4$ & $1,45 \pm 0,03$ & $S D$ & $S D$ & $0,060 \pm 0,023$ & $S D$ \\
\hline Gonzalo Moreno & $C V-31$ & $22,9 \pm 0,4$ & $5,44 \pm 0,01$ & $124 \pm 1$ & $2,4 \pm 0,1$ & $27,1 \pm 0,6$ & $1,3 \pm 0,1$ & $S D$ & $1,33 \pm 0,71$ & $S D$ & $0,49 \pm 0,02$ \\
\hline Gonzalo Moreno & $C V-32$ & $23,3 \pm 0,3$ & $4,85 \pm 0,01$ & $70 \pm 1$ & $2,57 \pm 0,01$ & $44 \pm 2$ & $1,14 \pm 0,02$ & $1,91 \pm 0,27$ & $S D$ & $1,03 \pm 0,014$ & $S D$ \\
\hline Gonzalo Moreno & $C V-29$ & $37,3 \pm 1,0$ & $8,4 \pm 0,1$ & $18 \pm 1$ & $2,33 \pm 0,05$ & $12,5 \pm 0,1$ & $1,1 \pm 0,05$ & $1,02 \pm 0,44$ & $S D$ & $0,22 \pm 0,006$ & $S D$ \\
\hline Gonzalo Moreno & MIR-16 & $24,5 \pm 0,6$ & $6 \pm 0,1$ & $8,4 \pm 0,1$ & $1,87 \pm 0,03$ & $14,4 \pm 0,1$ & $1,04 \pm 0,03$ & $1,66 \pm 0,99$ & $S D$ & $3,10 \pm 0,108$ & $0,13 \pm 0,07$ \\
\hline Gonzalo Moreno & MIR-20 & SD & $3,91 \pm 0,02$ & $S D$ & $2,13 \pm 0,03$ & $S D$ & $0,95 \pm 0,02$ & $S D$ & $S D$ & $S D$ & $0,74 \pm 0,12$ \\
\hline Gonzalo Moreno & $R V-25$ & $25,3 \pm 0,3$ & $16,3 \pm 0,4$ & $39 \pm 1$ & $5,7 \pm 0,2$ & $13,4 \pm 0$ & $0,88 \pm 0,02$ & $S D$ & $S D$ & $0,27 \pm 0,012$ & $S D$ \\
\hline Riberalta & $N A Z-3$ & $34,1 \pm 0,8$ & $8,7 \pm 0,1$ & $44,3 \pm 0,6$ & $6,3 \pm 0,2$ & $20,9 \pm 0,1$ & $4,66 \pm 0,01$ & $S D$ & SD & $0,07 \pm 0,047$ & $S D$ \\
\hline Riberalta & $N A Z-2$ & SD & $10,8 \pm 0,04$ & $S D$ & $6,9 \pm 0,2$ & $S D$ & $4,5 \pm 0,1$ & $S D$ & $0,94 \pm 0,14$ & $S D$ & $1,08 \pm 0,015$ \\
\hline Riberalta & SJ-12 & SD & $6,6 \pm 0,1$ & $S D$ & $3,14 \pm 0,04$ & $S D$ & $2,3 \pm 0,04$ & $S D$ & $2,83 \pm 2,0$ & $S D$ & $2,54 \pm 1,09$ \\
\hline Riberalta & SJ-15 & SD & $7,1 \pm 0,1$ & $S D$ & $3,5 \pm 0,1$ & $S D$ & $2,17 \pm 0,03$ & $S D$ & $2,13 \pm 0,42$ & $S D$ & $2,83 \pm 0,66$ \\
\hline Riberalta & $N A Z-7$ & SD & $5,2 \pm 0,1$ & $S D$ & $2,7 \pm 0,03$ & $S D$ & $1,66 \pm 0,01$ & $S D$ & $S D$ & $S D$ & $0,58 \pm 0,81$ \\
\hline Riberalta & $N A Z-8$ & SD & $6 \pm 0,1$ & $S D$ & $2,14 \pm 0,05$ & $S D$ & $1,45 \pm 0,01$ & $S D$ & $S D$ & $S D$ & $0,318 \pm 0,007$ \\
\hline Riberalta & SJ-11 & $47,1 \pm 0,5$ & $4,41 \pm 0,03$ & $39,1 \pm 0,2$ & $2,7 \pm 0,1$ & $29,1 \pm 0,4$ & $1,34 \pm 0,01$ & $6,91 \pm 0,92$ & $S D$ & $1,19 \pm 1,02$ & $S D$ \\
\hline Riberalta & $N A Z-4$ & $28,1 \pm 0,6$ & $5,57 \pm 0,04$ & $85,4 \pm 2,7$ & $2,45 \pm 0,03$ & $59 \pm 2$ & $1,32 \pm 0,02$ & $6,85 \pm 0$ & $0,53 \pm 0,02$ & $3,22 \pm 0,32$ & $0,41 \pm 0,021$ \\
\hline Riberalta & $N A Z-5$ & $78 \pm 1,5$ & $6,2 \pm 0,1$ & $73 \pm 2$ & $2,31 \pm 0,02$ & $63 \pm 1$ & $1,3 \pm 0,1$ & $1,72 \pm 0,10$ & $0,225 \pm 0$ & $3,95 \pm 0,25$ & $1,01 \pm 0,05$ \\
\hline Riberalta* & SJ-9* & $37,1 \pm 0,3$ & $5,7 \pm 0,2$ & $51,7 \pm 0,7$ & $1,98 \pm 0,03$ & $201,1 \pm 0,4$ & $1,28 \pm 0,01$ & $S D$ & $S D$ & $S D$ & $4,13 \pm 1,75$ \\
\hline Riberalta & $N A Z-6$ & SD & $11,1 \pm 0,1$ & $S D$ & $2,2 \pm 0,1$ & $S D$ & $1,23 \pm 0,03$ & $S D$ & $0,80 \pm 0,19$ & $S D$ & $1,65 \pm 0,20$ \\
\hline Riberalta* & SJ-10* & $46,7 \pm 1,2$ & $9,5 \pm 0,2$ & $61,3 \pm 9$ & $2,4 \pm 0,1$ & $222,6 \pm 0,3$ & $1,18 \pm 0,02$ & $S D$ & $7,6 \pm 0,4$ & $S D$ & $8,43 \pm 0,45$ \\
\hline Riberalta & SJ-14 & SD & $8,9 \pm 0,1$ & $S D$ & $2,48 \pm 0,02$ & $S D$ & $1,07 \pm 0,04$ & $S D$ & $2,65 \pm 0,07$ & $S D$ & $4,69 \pm 1,44$ \\
\hline Riberalta & $N A Z-1$ & SD & $13,3 \pm 0,1$ & $S D$ & $2,4 \pm 0,2$ & $S D$ & $1,02 \pm 0,04$ & $S D$ & $0,28 \pm 0,05$ & $S D$ & $1,04 \pm 0,016$ \\
\hline Riberalta & SJ-13 & $45,2 \pm 0,7$ & $5,2 \pm 0,2$ & $133,9 \pm 0,9$ & $1,71 \pm 0,04$ & $31,1 \pm 0,3$ & $0,81 \pm 0,03$ & $3,71 \pm 0,18$ & $S D$ & $2,84 \pm 0,15$ & $S D$ \\
\hline Baures & $S C-8$ & $43,2 \pm 1,1$ & $5,79 \pm 0,03$ & $54,9 \pm 0,8$ & $7,2 \pm 0,5$ & $25,1 \pm 0,5$ & $13,2 \pm 0,4$ & $4,20 \pm 0,20$ & $3,93 \pm 0,24$ & $3,94 \pm 0,19$ & $1,14 \pm 0,06$ \\
\hline Baures & $C T-13$ & $126,4 \pm 6,0$ & $3,73 \pm 0,03$ & $106 \pm 3$ & $7,1 \pm 0,1$ & $97 \pm 3$ & $13,9 \pm 0,4$ & $12,79 \pm 0,67$ & $S D$ & $5,78 \pm 0,29$ & $0,154 \pm 0,08$ \\
\hline Baures & $S P-29$ & $85,4 \pm 3,7$ & $3,15 \pm 0,03$ & $76,6 \pm 0,8$ & $2,06 \pm 0,01$ & $51,5 \pm 0,9$ & $1,05 \pm 0,01$ & $S D$ & $S D$ & $S D$ & $0,17 \pm 0,009$ \\
\hline Baures & ITV-23 & $90,2 \pm 4,8$ & $3,73 \pm 0,02$ & $83 \pm 2$ & $3,1 \pm 0,1$ & $59, \pm 4$ & $2,8 \pm 0,1$ & $S D$ & $1,24 \pm 0,064$ & $S D$ & $0,906 \pm 0,04$ \\
\hline San Ignacio Mojos & EAR_28 & $8,6 \pm 0,3$ & SD & $6,7 \pm 0,1$ & $S D$ & $6,8 \pm 0,1$ & $S D$ & $5,50 \pm 0,26$ & $S D$ & $4,37 \pm 0,22$ & $S D$ \\
\hline San Ignacio Mojos & SRA-8 & SD & $5,5 \pm 0,02$ & $S D$ & $2,5 \pm 0,1$ & $S D$ & $1,6 \pm 0,1$ & $S D$ & $1,48 \pm 0$ & $S D$ & $0,407 \pm 0,002$ \\
\hline San Ignacio Mojos & EAR_33 & $16,1 \pm 0,4$ & SD & $6 \pm 0,3$ & $S D$ & $6,5 \pm 0,1$ & $S D$ & $5,86 \pm 0,33$ & $S D$ & $8,20 \pm 0,42$ & $S D$ \\
\hline
\end{tabular}

Los valores son expresados como el promedio de 6 determinaciones en dos diferentes días \pm la desviación estándar

SD. Sin Datos. *Muestras ganadoras de premios internacionales

\section{REFERENCIAS}

1. Bazoberry, O., Salazar C., 2008, El cacao en Bolivia, una alternativa económica de base campesina indígena, Cipca, La Paz Bolivia, 35- 37.

2. Cala M., Vasquez A., García A., Martínez J.R., Stanshenke E., 2011, Estudio Comparativo por electroforesis capilar y Cromatografía liquida de alta Eficiencia de catequinas extraídas de cinco variedades de cacao colombiano, Rev. Académica colombiana, 34, 372379.

3. Sotelo, L., Alvis, B.A., Arrázola, G., 2015, Evaluación de epicatequina, teobromina y cafeína en cascaras de cacao (Theobroma Cacao L.), determinación de su capacidad antioxidante. Rev. Colombiana de Ciencias Hortícolas, 9 (1) 124 - 134.

4. Zapata, S., Tamayo A., Rojano B.A., 2013, Efecto de la fermentación sobre la actividad antioxidante de diferentes cacaos colombianos. Rev. Cubana de Plantas Medicinales, 391 - 404. 
5. Hassan O., Nasarudim, R., Lee, S.L., 2004, Extracts of cacao (Theobroma Cacao L.) leaves and their antioxidation potential, Food Chemestry, 86(1), 41-46.

6. Sanchez, J.G., 2014, Extracción y cuantificación de teobromina existente en las semillas de cuatro variedades de Cacao (Theobroma Cacao L.) producidas en la provincia del oro - Machala - Ecuador.

7. Bucheli, P., Rousseau, G., Alvarez, M., Selabi, M., 2001, Developmental variation of sugars, carboxylic acids, purine alkaloids, fatty acids and endoproteinas e activity during maturation of theobroma Cacao L. Seeds. Rev. Agricultural and Food Chemestry, 49(10) 5046-5051.

8. Romero, C., Zambrano, A., 2012, Análisis de azúcares en pulpa de cacao por colorimetría y electroforesis capilar. Rev. Científica UDO Agrícola, 12(4), 906- 913.

9. Vazquez-Ovando, A., Ovando Medina, I., Adriano-Annaya L., 2016, Alcaloides y '́polifenoles del cacao, mecanismos que regulan su biositesis y sus implicaciones en el sabor y aroma. Archivos latinoamericanos de nutrición, 66 (3) 239-253.

10. Tejeda L, Alavarado, J.A., Desbic. M., Peñariieta, J.M., Cardenas, O., Alvares, Mt., 2013, Genes relacionados en la biosíntesis de la composición de polifenoles de la colección de papas de color andino. Food Science \& Nutrition, 2(1), 46-57.

11. Chuquimia F., Alvarado J.A., Peñarrieta J.M., Bergenstahl, B., 2008, Determinación de la capacidad antioxidante y la cuantificación de compuestos fenólicos y flavonódicos de 4 especies vegetales, Revista Boliviana de Química, 25(1), 75-83.

12. Peñarrieta, J.M., Alvarado, J.A., Bergenstaht, B., Akesson, B.,2008, Total antioxidant capacity and content of flavonoids and other phenolic compunds in canihua (Chenopodium Pallidicaude) an Andean Pseuocereal, Molecular Nutrition Food Research, 52(6), 708-717.

13. Martínez, A.C., 2015. Determinación de Polifenoles Totales, Actividad Antioxidante y Antocianinas de Jugo de Murtilla Ugni molinae Turcz Obtenido por Condensación de Vapor. Tésis Universidad Austral de Chile, Valdivia- Chile.

14. Pallares, A., Estupiñan, M., Parea, J., Lopez, L., 2016 ,Impacto de la fermentación y secado sobre el contenido de polifenoles y capacidad antioxidante del clon de cacao CCN- 51. Revista Ion, 29(2), 7-21.

15. Sotero, V., Maco, M., Vela, J.,Marino, C., Davila, E., 2011. Evaluación de la actividad antioxidante y compuestos fenólicos en pulpa y semillas de cuatro frutales amazónicos de la familia Sterculiaceae. Revista de la Sociedad Quimica del Peru, 66-78.

16. Parea, J., Cadena, T., Ardila, J., 2009. El cacao y sus productos como fuente de antioxidantes: Efecto del Procesamiento, Articulos Cientificos,41, 128-134.

17. Quispe, L., 2013. Teobromina, cafeína y catequinas, en granos de cacao de pucacaca y huingoyacu e influencia del cacao ccn-51 y del beneficio. Repositorio Institucional de la Universidad Nacional Agraria de la Selva. Lima, Perú.

18. Chumacero, C., Durka, W., Tsharntke, T., Hensen, I., 2013, Gene flow and genetic diversity in cultivated and wild cacao ( Theobroma cacao ) in Bolivia, American Journal of Botany, 100(1), 1-9.

19. Fernández, R.,Mecias, F., Guzman, A., Peña, M., Medina, H., Casanova, L., Barrera, A., Nivela, P., 2012, Efecto del tipo y tiempo de fermentación en la calidad física y química del cacao (Theobroma cacao L.) Tipo Nacional, Ciencia y tecnologia, 5(12), 7-12.

20. Essien,J., West G., Alderson, P.G., Turker, G., 2008, Phenolic content and antioxidant capacity of hibrid variety cocoa beans, Food Chemestry, 108, 1155-1159.

21. Camu, N.,Winter, T., Kaddo, S., Takramma, J., 2008, Fermentation of cocoa beans: influence of microbial activities and polyphenol concentrations on the flavour of chocolate, The Science of food and Agriculture, 88, 2288-2297.

22. Loureiro, G., Araujo. Q., Valle, R., Andrade, G., Moreira de Souza, S., 2007, Influencia de factores agroambientales sobre la del clon de cacao (Theobroma Cacao L.) PH- 16 en la región Cacaotera de Bahia, Brasil, Ecosistemas y Recursos Agropecuarios 4(12), 579587.

23. Hansen, C., Del Olmo, M., Burri, C., 1998, Ensyme Activities in Cocoa Beans During Fermentation, Sciencies Food Agricolas, 77 273-281. 